\title{
DEVELOPMENT AND VALIDATION OF A HIGH CONSTRAINT MODIFIED BOUNDARY LAYER FINITE ELEMENT MODEL
}

\author{
M. Verstraete ${ }^{1}$, W. De Waele ${ }^{1}$, S. Hertelé $e^{1,2}$ \\ ${ }^{1}$ Ghent University, Laboratory Soete, Department of Mechanical Construction and Production, Belgium \\ ${ }^{2}$ FWO Flanders (Research Foundation Flanders) aspirant
}

\begin{abstract}
When a notched structure is loaded, its behaviour is not only affected by the material properties but also by the geometry (of both the structure and the defect) and loading condition, alternatively termed as constraint condition. Therefore, the relation between the failure behaviour of a small scale fracture mechanics test and a full scale structure needs to be elucidated.

In an attempt to understand and describe such relationships, the crack tip stress fields are analysed by means of finite element simulations and compared for several test specimen geometries. A reference for comparison is the crack tip stress field obtained from a high constraint reference geometry, further called a modified boundary layer model.

First, this article provides some theoretical background on the modified boundary layer model. Second, the development of a $2 \mathrm{D}$ model is outlined in detail, focussing on the mesh design in the vicinity of the crack tip and the applied boundary conditions. Afterwards, an analytical and numerical validation is provided, based on the level of the applied load and, on the other hand, on the magnitude of the crack tip stress fields. Finally, this validated model is used for the comparison of several constraint parameters. This comparison indicates a weak influence of the $T$-stress on the Q-parameter for positive $T$-stresses. In contrast, negative $T$-stresses result in more pronounced negative Q-values.
\end{abstract}

Keywords: Constraint, J-Q theory, crack tip stress fields, validation

\section{INTRODUCTION}

\subsection{General}

Since the 1920s researchers try to understand the failure behaviour of cracked structures. It has quickly been understood that the shape and magnitude of the stress fields ahead of the crack tip influence this behaviour. Consequently, theoretical concepts have been developed to describe the stress fields ahead of the crack tip. At first, materials have been studied in which hardly any plasticity developed in the vicinity of the crack tip. These studies have resulted in the linear elastic fracture mechanics (LEFM) theory. Within this theoretical concept, the crack tip stress fields are described by an analytical expression developed by Williams in the late 1950s [1].

$$
\sigma_{i j}=\frac{K_{I}}{\sqrt{2 \pi r}} f_{i j}(\theta)+\left[\begin{array}{ccc}
T & 0 & 0 \\
0 & 0 & 0 \\
0 & 0 & v T
\end{array}\right]+\cdots
$$

In this so-called Williams expansion, the first term was believed to be dominant over the others. As a result, LEFM theory only considers $K_{I}$, defining the magnitude of the first term, to describe the crack tip stress fields. Unfortunately, this theory breaks down when the material ahead of the crack tip deforms plastically. To overcome this shortcoming, the elastic plastic fracture mechanics (EPFM) theory has been developed by Hutchinson, Rice and Rosengren (HRR) [2]. They described the crack tip stress fields by means of the following equation, known as the HRR-solution:

$$
\sigma_{\mathrm{ij}}=\sigma_{0}\left(\frac{E J}{\alpha \sigma_{0}^{2} I_{\mathrm{n}} \mathrm{r}}\right)^{1 / \mathrm{n}+1} \widetilde{\sigma}_{\mathrm{ij}}(\mathrm{n}, \theta)
$$


The above description works well for situations where the plasticity is limited to a small zone in the vicinity of the crack tip, alternatively termed as small scale yielding conditions. Under these conditions, both the shape and magnitude of the crack tip stress field remain geometry independent. In contrast, when fully contained yielding conditions are examined, it is not longer possible to accurately describe the crack tip stress fields by means of a single parameter. Under these conditions the material's fracture toughness depends on the specimen's geometry and the loading condition $[3,4]$. This dependence is alternatively termed as the constraint condition. Consequently, fracture toughness results, in terms of critical $J$ or $K_{I}$ values, are no longer directly transferrable between a laboratory scale specimen and a large structure.

\subsection{Constraint parameters}

In an attempt to clarify the influence of different constraint conditions, several new theoretical concepts have been developed. At first, the LEFM theory has been extended, also considering the second term in the Williams expansion. This term's magnitude is defined by the level of the $T$-stress. As such, a two parameter theoretical concept was developed, known as the $K$ - $T$ theory [5-7]. However, as already stated in the previous paragraph, this theory is only applicable for situations that lack plasticity in the vicinity of the crack tip.

Nowadays, the most suitable theory for investigating fully contained yielding conditions is an extension of the $J$-integral concept. This extension has been developed in the early 1990 s by O'Dowd \& Shih and describes the constraint effects by means of the Q-parameter $[8,9]$. This parameter has been defined by the following relationship:

$$
\sigma_{\mathrm{ij}}=\left(\sigma_{\mathrm{ij}}\right)_{\mathrm{HRR}}+\mathrm{Q} \sigma_{0} \delta_{\mathrm{ij}}
$$

It is clear that the leading term is still the HRR-solution, setting the size scale of the crack tip stress field. Additionally, the Q-parameter defines the magnitude of a uniform hydrostatic shift in the crack tip stress field. This shift will lead to a lowered stress level for negative $Q$-values, representing low constraint conditions. In contrast, an increased stress level (positive Q) will be observed for high constraint conditions. O'Dowd \& Shih have shown that the expression in Eq.3 is valid for distances $r$ ahead of the crack tip between $\mathrm{J} / \sigma_{0}$ and $5 \mathrm{~J} / \sigma_{0}$. As a result, the Q-parameter is most often evaluated at a distance $\mathrm{r}=2 \mathrm{~J} / \sigma_{0} . \ln$ that case, a simple modification of Eq.3 defines the Q-parameter:

$$
\mathrm{Q}=\frac{\sigma_{\mathrm{ii}}-\left(\sigma_{\mathrm{ii}}\right)_{\text {HRR }}}{\sigma_{0}} \quad \text { evaluated at: } \mathrm{r}=2 \mathrm{~J} / \sigma_{0}
$$

This initial definition, provided by O'Dowd \& Shih, is based on the HRR solution as the reference field. However, nowadays applications rather use the small scale yielding (SSY) reference stress field to calculate the Q-parameter.

$$
\sigma_{\mathrm{ij}}=\left(\sigma_{\mathrm{ij}}\right)_{\mathrm{SSY} ; \mathrm{T}=0}+\mathrm{Q} \sigma_{0} \delta_{\mathrm{ij}}
$$

This field is used because of its good correspondence with the HRR-field in terms of shape and magnitude. Furthermore, the SSY field can be more easily calculated, based on a modified boundary layer (MBL) analysis. This analysis requires a MBL model, of which the development and a validation are presented in this paper.

The remainder of this paper is organized as follows. First, some more detailed theoretical background is provided in the $2^{\text {nd }}$ paragraph. Afterwards, an analytical and numerical validation is provided in the $3^{\text {rd }}$ paragraph. Finally, paragraph 4 describes a comparison between the $T$-stress and Q-parameter based on the validated model. 


\section{THE MODIFIED BOUNDARY LAYER MODEL}

\section{$2.1 \quad$ General}

A modified boundary layer model aims to generate a high constraint stress field in the vicinity of the crack tip, representing small scale yielding conditions (see §1.2). These conditions require a situation of plain strain. In addition, the stress field around the crack tip should be dominated by the $1 / \sqrt{r}$ singularity in accordance to the LEFM theory. This stress field can be obtained from the finite element simulations of a circular body around the crack tip, schematically represented in Figure 1.

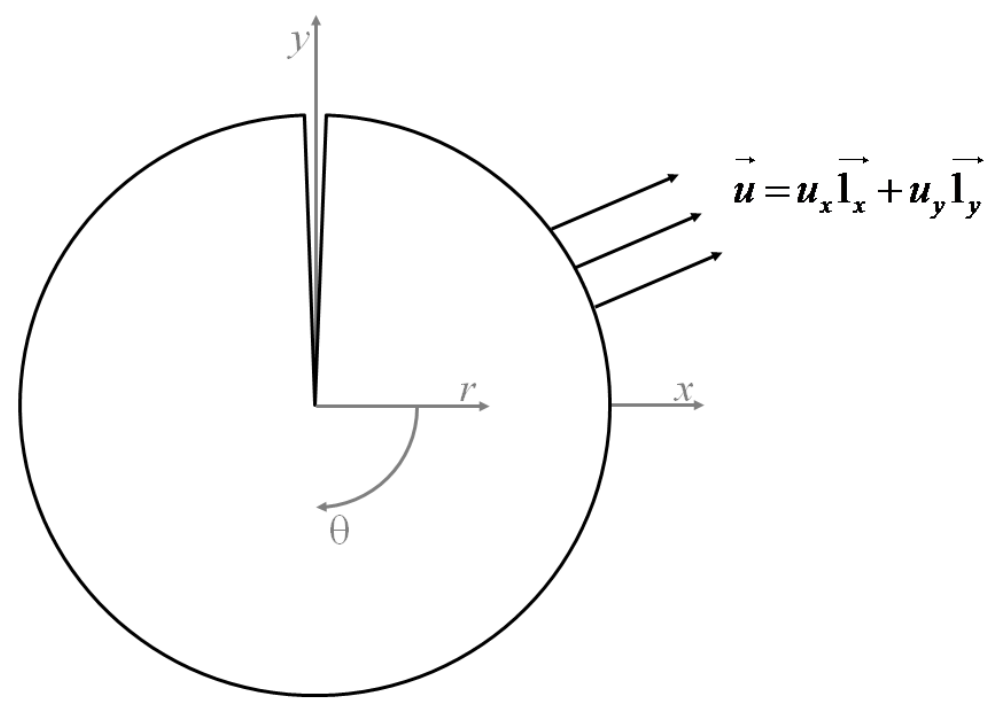

Figure 1: Schematic representation of Modified Boundary Layer model

A displacement of the elastic mode I singular field is imposed on the boundaries of this body. This displacement field is defined by the following equations:

$$
\begin{aligned}
& u_{x}=\mathrm{K}_{\mathrm{I}} \frac{1+\mathrm{v}}{\mathrm{E}} \sqrt{\frac{\mathrm{r}}{2 \pi}} \sin \left(\frac{\vartheta}{2}\right)(3-4 \mathrm{v}-\cos ((9)) \\
& u_{y}=-\mathrm{K}_{\mathrm{I}} \frac{1+\mathrm{v}}{\mathrm{E}} \sqrt{\frac{\mathrm{r}}{2 \pi}} \cos \left(\frac{\vartheta}{2}\right)(3-4 \mathrm{v}-\cos ((9))
\end{aligned}
$$

The magnitude of the above displacement fields is controlled by $K_{I}$. However, since LEFM theory applies, $K_{I}$ can also be expressed in terms of $J$. For plane strain conditions, the following relationship applies:

$$
J=\frac{K_{I}^{2}\left(1-v^{2}\right)}{E}
$$

Apart from the plane strain conditions described so far, the MBL model can also be used to study the effect of other constraint conditions on the magnitude of the crack tip stress field. These constraint conditions are created by applying different $T$-stress levels on the boundary of the MBL FE model:

$$
\begin{aligned}
& u_{x, T}=-\mathrm{T} \frac{\mathrm{v}(1+\mathrm{v})}{\mathrm{E}} \mathrm{r} \sin (\vartheta) \\
& u_{y, T}=-\mathrm{T} \frac{1-\mathrm{v}^{2}}{\mathrm{E}} \mathrm{r} \cos (\vartheta)
\end{aligned}
$$




\subsection{Finite element model}

A parametric Python ${ }^{\circledR}$ script was developed to generate the model and the input file needed for the Abaqus ${ }^{\circledR}$ processor. This model will be used to study the constraint effects for cracks obtained after fatigue pre-cracking. Hence, an infinitely sharp crack should be modelled. Unfortunately, the corresponding mesh configuration will neither be able to withstand large deformations nor will such mesh yield realistic stress distributions. Consequently, an initially blunted crack is modelled with a notch tip radius $\left(r_{0}\right)$ of $2.5 \mu \mathrm{m}$. This radius can be assumed to represent an infinitely sharp crack $[10,11]$. Furthermore, the outer radius is $10^{6}$ times the notch tip radius, which is in good correspondence with reported MBL models $[10,12,13]$. This large outer radius prevents the interaction of plasticity effects with the applied linear elastic boundary conditions.

In order to obtain the required plane strain boundary conditions, the developed FE model is a 2D model that consists of plane strain linear elements with reduced integration (Abaqus ${ }^{\circledR}$ type CPE4R). In addition, a gradually coarsening mesh is used to minimize the number of elements. This mesh consists of 210 rings of 80 elements around the circumference (see Figure 2a). Furthermore, the radial size of the smallest elements is equal to $r_{0} / 10$ (see Figure $2 \mathrm{~b}$ ) to accurately describe the stress field near the crack tip. The final mesh contains 16638 elements. However, it should be noted that the minimum number of elements needed for an accurate description of the stress fields has not yet been thoroughly investigated.

a)

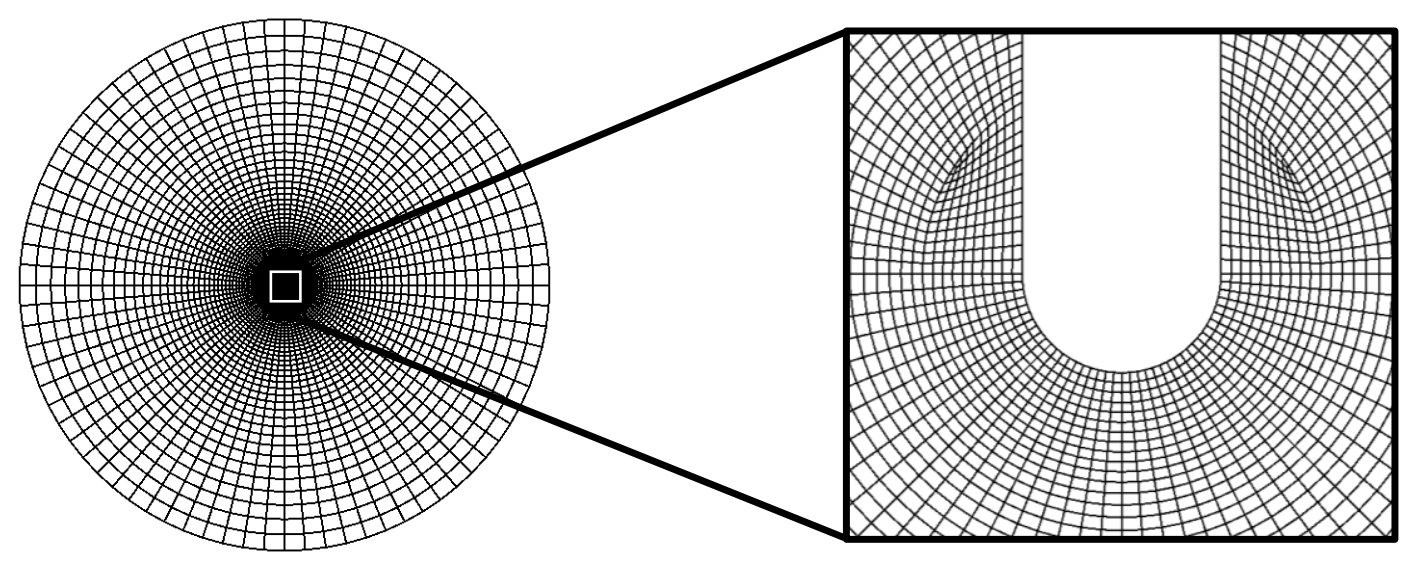

Figure 2: Mesh configuration of MBL model: global view (a) and detailed view of the crack tip (b)

\subsection{Evaluation of boundary conditions and applied load}

On the outer boundary of the model, the displacement fields described by Eqs.6-7 are applied. These fields are defined by means of continuously varying functions in terms of $r$ and $\theta$. In this case, $r$ equals the outer radius of the MBL model and the angle is varied between $\theta=0$ and $\theta=2 \pi$. To evaluate the accuracy of the applied boundary conditions, the resulting displacement fields are compared to the theoretical displacement fields (Figure 3). An excellent correspondence is observed.

a)

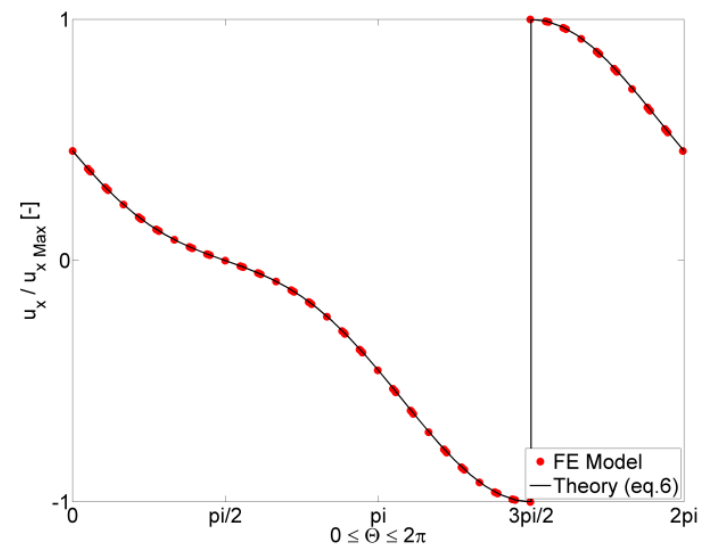

b)

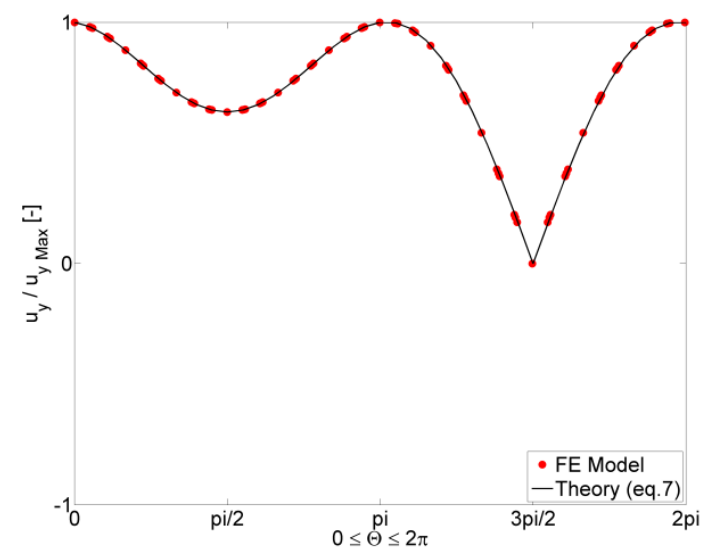

Figure 3: Applied displacement boundary conditions in $\mathrm{x}$-direction (a) and y-direction (b) 
Considering the above correspondence, the calculated load level should correspond to the applied load level. Therefore, a comparison is made between the applied and calculated $J$-value. A $J$-level of $1000 \mathrm{~N} / \mathrm{mm}$ is applied. A limited difference of $3 \%$ is noted. However, a comparable difference has also been reported in literature [14]. Therefore, this correspondence is considered satisfying.

\section{VALIDATION OF CRACK TIP STRESS FIELDS}

\subsection{Linear elastic validation}

At first the MBL FE model is validated for a linear elastic material, corresponding to LEFM theory. In such case, the shape of the crack tip stress field should match the theoretical shape described by the Williams expansion (eq.1). For an applied $T$-stress level equal to zero, this equation reduces to the expression below for the crack tip opening stress, $\sigma_{x x}$, ahead of the crack tip:

$$
\sigma_{x x}=\frac{K_{I}}{\sqrt{2 \pi r}}
$$

This theoretical distribution is plotted in Figure 4, also presenting the stress distribution obtained from the FE model. An excellent correspondence is observed between both distributions.

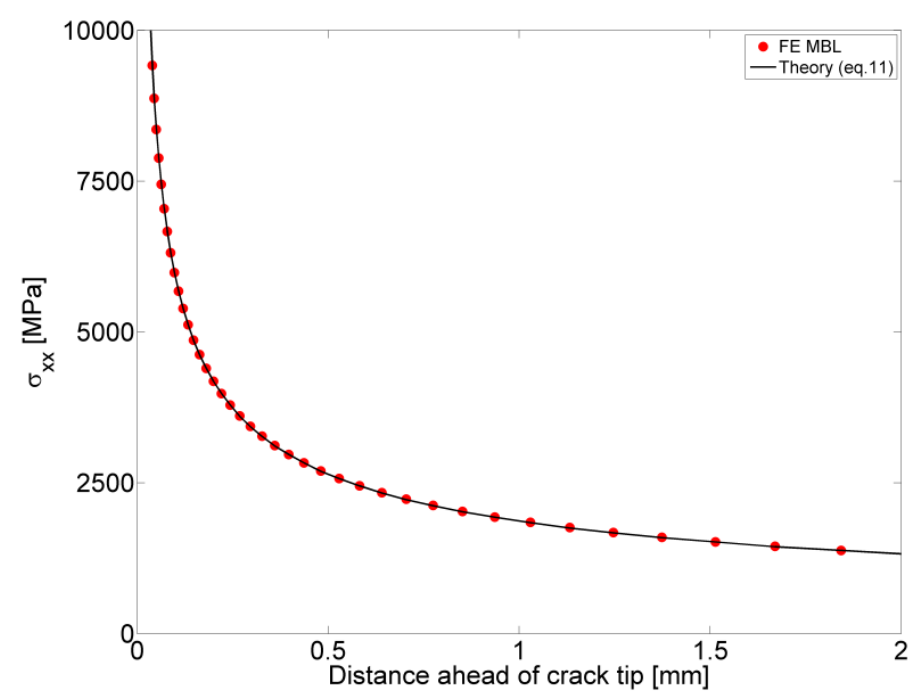

Figure 4: Linear elastic validation of MBL crack tip stress fields (at $\theta=\pi / 2$ )

\subsection{Elastic-plastic validation}

For a second validation, the plasticity effects around the crack tip are taken into account. Unfortunately, no theoretical solution exists for these SSY crack tip stress fields. Therefore, the results from the MBL FE model are compared to the results obtained from a literature review [8, 15]. For this validation a Ramberg-Osgood stress-strain relationship is assumed:

$$
\varepsilon=\frac{\sigma}{\mathrm{E}}+\alpha\left(\frac{|\sigma|}{\sigma_{0}}\right)^{\mathrm{n}-1} \frac{\sigma}{\mathrm{E}}
$$

For the validation, the following values are considered: Young's modulus $E=200 \mathrm{GPa}$, yield strength $\sigma_{0}=400 \mathrm{MPa}, \alpha=1$ and strain hardening exponent $n=10$. Furthermore, small strain conditions are assumed (NIGeom has been set off in Abaqus ${ }^{\circledR}$ ).

The crack tip stress field was studied for an applied load level $J=1000 \mathrm{~N} / \mathrm{mm}$ and a root radius of $2.5 \mu \mathrm{m}$. The resulting stress field $\sigma_{x x}$ is plotted in Figure 5. Comparing this stress field with the results from literature, a fairly good correspondence is achieved. Therefore, the MBL FE model is considered to correctly predict the crack tip stress fields. 


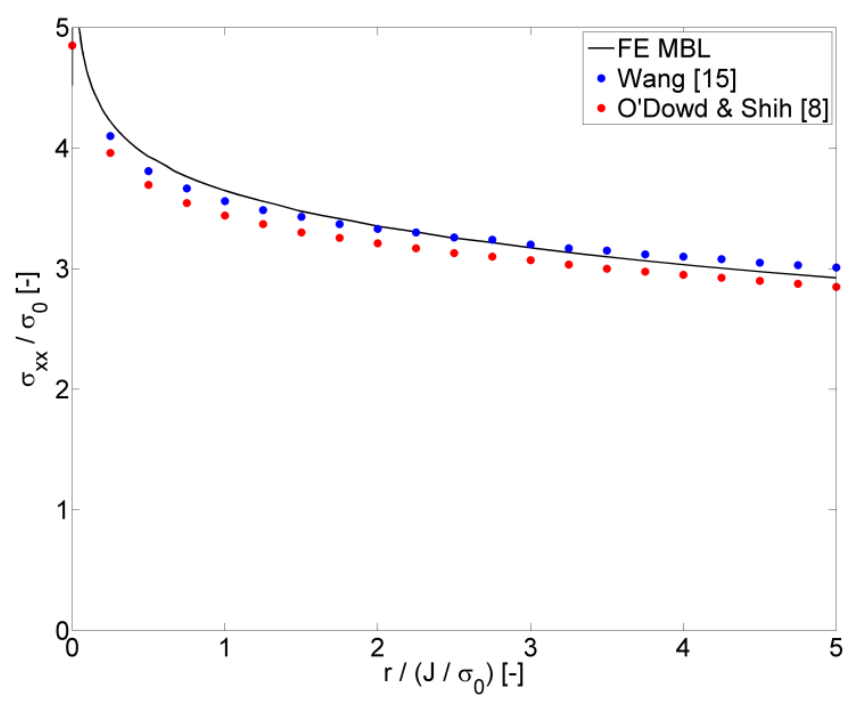

Figure 5: Elastic-plastic validation of MBL crack tip stress fields (at $\theta=\pi / 2$ )

\section{CONSTRAINT}

As already briefly described in $\S 2.1$, the MBL FE model is a useful tool to investigate the influence of different constraint conditions in terms of $T$-stress. This paragraph first investigates the exact influence of the $T$-stress on the magnitude and shape of the crack tip stress fields. Second, the Q-stress is calculated for each of the studied $T$-stress levels. These calculations lead to a relationship between the $T$-stress and Q-parameter.

\subsection{Influence of $T$-parameter on crack tip stress field}

Based on eq.9-10 an extra displacement can be applied on the outer boundary of the MBL model. This will generate different constraint conditions in the vicinity of the crack tip, most likely resulting in a shift of the crack tip stress field. This shift is expected towards higher stress levels for high constraint conditions (positive $T$-stresses). In contrast, lower stress levels are expected for so-called low constraint conditions (negative $T$-stresses). Indeed, as indicated by Figure 6, a positive $T$-stress results in a higher stress $\sigma_{x x}$, whereas a negative $T$-stress results in a lower stress $\sigma_{x x}$.

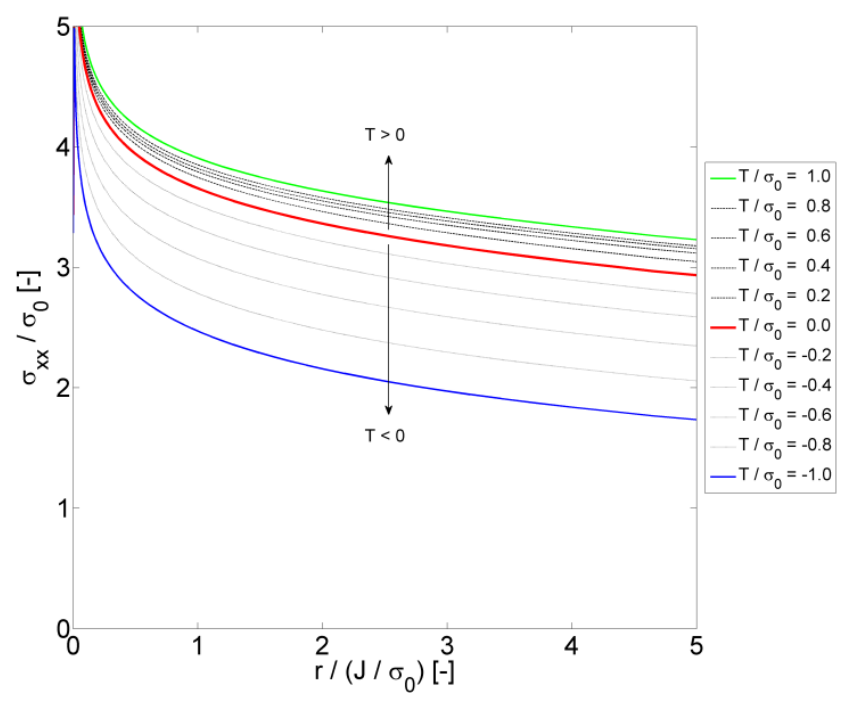

Figure 6: Influence of $T$-stress on the stress level ahead of the crack tip (at $\theta=\pi / 2$ ) 


\subsection{Relationship between $\boldsymbol{T}$-stress and Q-parameter}

From the above crack tip stress fields, the Q-parameter is calculated for different $T$-stress levels. Consequently, a relationship between these two constraint parameters is obtained. This relationship is displayed in Figure 7 . When the results from the MBL FE model are compared to results published in literature [15], an excellent correspondence is achieved. Only for the smallest investigated $T$-stress level a minor difference is observed.

From the obtained relationship it can be concluded that positive $T$-stress levels result in limited constraint conditions if expressed by the Q-parameter. In contrast, negative $T$-stress levels tend to strongly influence the Q-parameter. These results are in correspondence with the results of O'Dowd \& Shih [9].

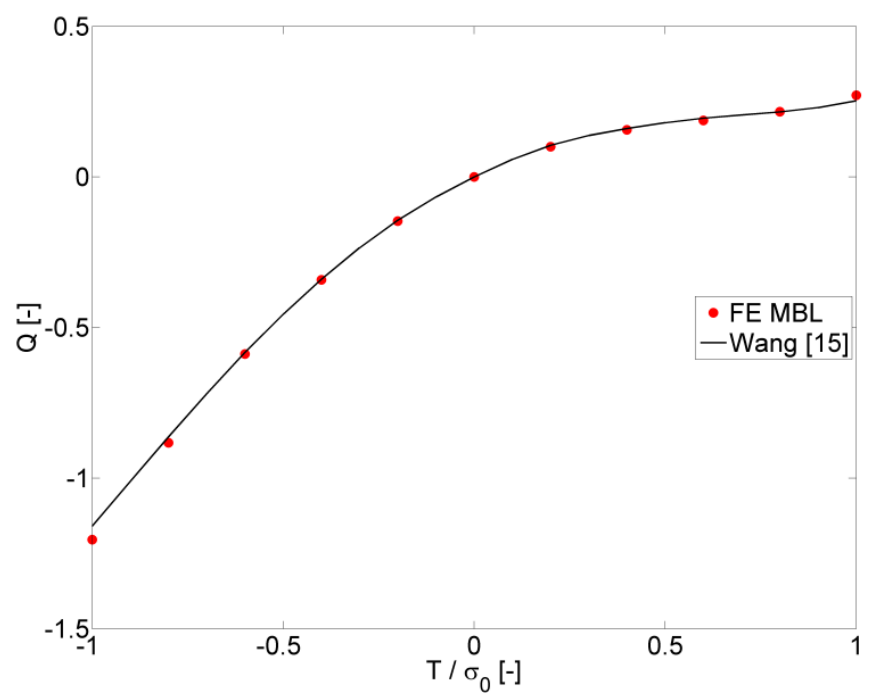

Figure 7: Relationship between $T$-stress and Q-parameter obtained from MBL analysis 


\section{CONCLUSIONS}

A finite element model was presented to calculate the magnitude of the crack tip stress fields in a MBL model under different constraint conditions. The following conclusions were drawn:

- The relationship between the Q-parameter and $T$-stress was investigated, showing a weak influence of the $T$-stress on the Q-parameter for positive $T$-stresses. In contrast, negative $T$-stress resulted in pronounced negative $Q$-values.

- For elastic-plastic conditions the obtained stress field showed an excellent correspondence to the stress fields published by other researchers.

- For linear elastic conditions, the crack tip stress fields corresponded to the analytical crack tip stress field.

- A limited difference was observed between the applied load level in terms of $J$ and the calculated load level.

\section{NOMENCLATURE}

$\begin{array}{lll}f_{i j}(\vartheta) & \text { Angular function } & {[-]} \\ \vartheta & \text { Angular position centered at the crack-tip } & {[-]} \\ Q & \text { Constraint parameter } & {[-]} \\ \tilde{\sigma}_{i j}(n, \vartheta) & \text { Dimensionless functions } & {[-]} \\ \alpha, n & \text { Dimensionless Ramberg-Osgood parameters } & {[-]} \\ u_{x} & \text { Displacement in x-direction } & {[\mathrm{mm}]} \\ u_{y} & \text { Displacement in y-direction } & {[\mathrm{mm}]} \\ r & \text { Distance ahead of the crack-tip } & {[\mathrm{mm}]} \\ J & \text { Energy release rate } & {[\mathrm{N} / \mathrm{mm}]} \\ I_{n} & \text { Integration constant depending on } n & {[-]} \\ \delta_{i j} & \text { Kronecker delta } & {[-]} \\ K_{I} & \text { Mode I stress intensity factor } & {[\mathrm{MPa} \sqrt{ } \mathrm{mm}]} \\ r_{0} & \text { Notch tip radius } & {[\mathrm{mm}]} \\ v & \text { Poisson coefficient } & {[-]} \\ \sigma_{i j} & \text { Stress component in the vicinity of the crack tip } & {[\mathrm{MPa}]} \\ \varepsilon & \text { True strain } & {[-]} \\ \sigma & \text { True stress } & {[\mathrm{MPa}]} \\ T & T \text {-stress } & {[\mathrm{MPa}]} \\ \sigma_{0} & \text { Yield stress of the material } & {[\mathrm{MPa}]} \\ E & \text { Young's modulus } & {[\mathrm{MPa}]}\end{array}$

\section{ACKNOWLEDGEMENTS}

The authors would like to acknowledge the financial support of the IWT (Agency for innovation by science and technology - grant $\mathrm{n}^{\circ}$ SB-091512) and the FWO (Research Foundation Flanders - grants $\mathrm{n}^{\circ}$ 1.1.880.09.N and 1.5.247.08N.00). 


\section{REFERENCES}

1. Williams, M.L., On the stress distribution at the base of a stationary crack. Journal of Applied Mechanics, 1957. 24: p. 109-114.

2. Anderson, T.L., Fracture Mechanics - Fundamentals and Applications. 1995.

3. $\mathrm{Xu}$, J., et al., Effects of embedded defects in pipelines subjected to plastic strains during operation. International Journal of Pressure Vessels and Piping, 2009. 86: p. 787-797.

4. Thaulow, C., et al., Constraint correction of high strength steel - selection of test specimens and applications of direct calculations. Engineering Fracture Mechanics, 2004. 71: p. 2417-2433.

5. Du, Z.Z. and J.W. Hancock, The effect of non-singular stresses on crack-tip constraint. Journal of Mechanics and Physics of solids, 1991. 39: p. 555-567.

6. Al-Ani, A.M. and J.W. Hancock, J-dominance of short crack in tension and bending. Journal of Mechanics and Physics of solids, 1991. 39: p. 23-43.

7. Betegon, C. and J.W. Hancock, Two-parameter characterization of elastic-plastic crack tip fields. ASME Journal of applied mechanics, 1991. 58: p. 104-113.

8. O'Dowd, N.P. and C.F. Shih, Family of crack-tip fields characterized by a triaxiality parameter - Part I. structure of fields. Journal of Mechanics and Physics of solids, 1991. 99: p. 989-1015.

9. O'Dowd, N.P. and C.F. Shih, Family of crack-tip fields characterized by a triaxiality parameter - Part II. Fracture Applications. Journal of Mechanics and Physics of solids, 1992. 40: p. 939-963.

10. Cravero, S. and C. Ruggieri, $A$ two-parameter framework to describe the effect of constraint loss on cleavage fracture and implications for failure assessments of cracked components. Journal of Brazilian Society of Mechanical Sciences and Engineering, 2003. 15: p. 403-412.

11. Ranestad, O., Z.L. Zhang, and C. Thaulow, Quantification of geometry and material mismatch constraint in steel weldments with fusion line cracks. International Journal of Fracture, 1999. 99: $p$. 211-237.

12. Horn, A.J., Development of an engineering assessment procedure for predicting cleavage fracture from non-sharp defects using the failure assessment diagram, in School of Materials, University of Manchester, 2010, PhD-Thesis.

13. Sherry, A.H., et al., Material constraint parameters for the assessment of shallow defects in structural components - Part I. Parameter solutions. Engineering Fracture Mechanics, 2005. 72: p. 2373-2395.

14. Zhang, Z.L., M. Hauge, and C. Thaulow, The Effect of $T$ stress on the near tip stress field on an elastic-plastic interface crack, in International conference on fracture. 1997: Sydney, Australia.

15. Wang, X., Two-parameter characterization of elastic-plastic crack front fields: surface cracked plates under tensile loading. Engineering Fracture Mechanics, 2009. 76: p. 958-982. 